\title{
Quantum Evolution of the Bianchi Type I Model
}

\author{
V.N. Folomeev and V.Ts. Gurovich ${ }^{1}$ \\ Physics Institute of NAN KR, \\ 265 a, Chui str., Bishkek, 720071, Kyrgyz Republic.
}

November 21, 2018

${ }^{1}$ email: gurovich@grav.freenet.bishkek.su 


\begin{abstract}
The behaviour of the flat anisotropic model of the Universe with a scalar field is explored within the framework of quantum cosmology. The principal moment of the account of an anisotropy is the presence either negative potential barrier or positive repelling wall. In the first case occur the above barrier reflection of the wave function of the Universe, in the second one there is bounce off a potential wall. The further evolution of the Universe represents an exponential inflating with fast losses of an anisotropy and approach to the standard cosmological scenario.
\end{abstract}




\section{Introduction}

One of the basic problems of the standard cosmological scenario is the presence of an initial singularity. For its elimination are used the various approaches, one of which is a quantum cosmology originated by DeWitt [1] more than thirty years ago. In last one the whole Universe is described with the help of the wave function. Until recently main part of papers was devoted to the consideration of the process of quantum creation of the closed Universe. In this direction by number of authors [2] was developed the scenario of the spontaneous creation of the closed Universe from "nothing", where "nothing" means the state with absence not only matter, but also the spacetime in classical understanding. Thus it is necessary to note that the closed Universe has a zero total energy and charge therefore there is no violation of any conservation laws. At describing of process of creation of the Universe the so-called instanton method [3] is widely used. In the last one the closure of the Universe is a necessary requirement of the quantum creation, otherwise the action is equal to infinity and, hence, relevant probability of the creation is near to zero. However, recently observant data have appeared according to which our Universe probably is open. A number of models of the quantum nucleating of the open Universe were proposed in this connection. One of its is the Hawking-Turok instanton [4], for which the opportunity of an analytic continuation of the instanton solution not only in the direction of creation of the closed Universe, but also in the direction of the open Universe is shown. This result, being interesting on itself, is not unexpected. For a long time it is known (see e.g. [5]) that dividing by various ways the four-dimensional de Sitter Universe on space and time we can obtain the various de Sitter Universes: closed, flat or open. The quantum creation of the open Universe can seem not actual in view of the noted above infinite value of action. But here it is possible to remember the theory of nucleation of the open Universe from bubble [6,7] filled by false vacuum, when the Universe inside of this bubble looks finite from the point of view of an external observer and open, that is infinite, from the point of view of an inside observer. Therefore, for an external observer the process of the instanton nucleation of the open Universe is quite possible and the growth of the bubble size is carried out by transferring of energy from the surrounding de Sitter space.

\section{Basic classical equations}

We shall consider an anisotropic Bianchi type I model. For it the synchronous form of metric can be expressed as (velocity of light is equal 1):

$$
d s^{2}=d t^{2}-a_{1}^{2}(t) d x^{2}-a_{2}^{2}(t) d y^{2}-a_{3}^{2}(t) d z^{2},
$$


where by $a_{1}, a_{2}$ and $a_{3}$ the scale factors on directions $x, y$, and $z$ accordingly are designated. This model is an anisotropic generalization of the Friedmann model with Euclidean spatial geometry. Three scale factors $a_{1}, a_{2}$ and $a_{3}$ are determined via Einstein's equations. For convenience of realization of the analytical calculations we can write them as follows [8] :

$$
a_{1}=r(t) q_{1}, \quad a_{2}=r(t) q_{2}, \quad a_{3}=r(t) q_{3},
$$

where $q_{1}, q_{2}, q_{3}$ are dimensionless variable subordinated to the following requirements:

$$
\prod_{\alpha=1}^{3} q_{\alpha}=1, \quad \sum_{\alpha=1}^{3}\left(\dot{q}_{\alpha} / q_{\alpha}\right)=0
$$

(dot means derivative with respect to time $t$ ); whence follows that $\prod_{\alpha=1}^{3} a_{\alpha}=r^{3}$.

For the line element (11) with the account of (2) the components of the Ricci tensor write as:

$$
\begin{aligned}
-R_{0}^{0} & =3 \frac{\ddot{r}}{r}+\sum_{\alpha=1}^{3}\left(\frac{\dot{q}_{\alpha}}{q_{\alpha}}\right)^{2}, \\
-R_{\alpha}^{\alpha} & =\frac{\ddot{r}}{r}+2\left(\frac{\dot{r}}{r}\right)^{2}+3 \frac{\dot{r}}{r} \frac{\dot{q}_{\alpha}}{q_{\alpha}}+\left(\frac{\dot{q}_{\alpha}}{q_{\alpha}}\right)^{\cdot}, \\
-R & =6\left[\frac{\ddot{r}}{r}+\left(\frac{\dot{r}}{r}\right)^{2}\right]+\sum_{\alpha=1}^{3}\left(\frac{\dot{q}_{\alpha}}{q_{\alpha}}\right)^{2} .
\end{aligned}
$$

Let's use the last one for obtaining (1-1) and (2-2) components of the Einstein tensor:

$$
\begin{aligned}
& G_{1}^{1}=2 \frac{\ddot{r}}{r}+\left(\frac{\dot{r}}{r}\right)^{2}-3 \frac{\dot{r}}{r} \frac{\dot{q}_{1}}{q_{1}}-\left(\frac{\dot{q}_{1}}{q_{1}}\right)^{\cdot}+\frac{1}{2} \sum_{\alpha=1}^{3}\left(\frac{\dot{q}_{\alpha}}{q_{\alpha}}\right)^{2}, \\
& G_{2}^{2}=2 \frac{\ddot{r}}{r}+\left(\frac{\dot{r}}{r}\right)^{2}-3 \frac{\dot{r}}{r} \frac{\dot{q}_{2}}{q_{2}}-\left(\frac{\dot{q}_{2}}{q_{2}}\right)^{\cdot}+\frac{1}{2} \sum_{\alpha=1}^{3}\left(\frac{\dot{q}_{\alpha}}{q_{\alpha}}\right)^{2} .
\end{aligned}
$$

Subtracting from $G_{1}^{1}$ the component $G_{2}^{2}$ one obtains:

$$
3 \frac{\dot{r}}{r}\left(\frac{\dot{q}_{2}}{q_{2}}-\frac{\dot{q}_{1}}{q_{1}}\right)+\left(\frac{\dot{q}_{2}}{q_{2}}-\frac{\dot{q}_{1}}{q_{1}}\right)^{\cdot}=0 .
$$

Entering in the last equation the notification $Q_{\alpha \beta}=\left(\dot{q}_{2} / q_{2}-\dot{q}_{1} / q_{1}\right)$ let's have:

$$
3 \frac{\dot{r}}{r}+\frac{\dot{Q}_{\alpha \beta}}{Q_{\alpha \beta}}=0
$$


that after integration gives:

$$
Q_{\alpha \beta}=C_{\alpha \beta} / r^{3}
$$

where $C_{\alpha \beta}$ are integration constants. From here we get:

$$
\frac{\dot{q}_{\alpha}}{q_{\alpha}}=\frac{C_{\alpha}}{r^{3}}
$$

and according to requirements (3), $\sum_{\alpha=1}^{3} C_{\alpha}=0$. Thus integrating the last equation one finds:

$$
q_{\alpha}=A_{\alpha} \exp \left\{C_{\alpha} \int \frac{d t}{r^{3}}\right\}
$$

where $A_{\alpha}$ are integration constants and $\prod_{\alpha=1}^{3} A_{\alpha}=1$. Now, using the relation (5), from (\#) is got:

$$
\begin{aligned}
-R_{0}^{0} & =3 \frac{\ddot{r}}{r}+\frac{1}{r^{6}} \sum_{\alpha=1}^{3} C_{\alpha}^{2} \\
-R & =6\left[\frac{\ddot{r}}{r}+\left(\frac{\dot{r}}{r}\right)^{2}\right]+\frac{1}{r^{6}} \sum_{\alpha=1}^{3} C_{\alpha}^{2},
\end{aligned}
$$

$\sum_{\alpha=1}^{3} C_{\alpha}^{2}$ determines an anisotropy of the given model.

\section{Quantum evolution of the Universe}

The possibility of the nucleation of an open Universe circumscribed in Introduction gives the basis for the consideration of the quantum creation of a flat Universe. As is known the basic equation of the quantum cosmology is the Wheeler-DeWitt (WDW) equation. For its making we shall consider the theory of a scalar field $\varphi$ with Lagrangian

$$
L=-R / 2+\left(\partial_{\mu} \varphi\right)^{2} / 2-V(\varphi)
$$

or using the expression for scalar curvature $R$ from (身) one obtains

$$
L=-3 r \dot{r}^{2}+\frac{r^{3}}{2} \sum_{\alpha=1}^{3}\left(\frac{\dot{q}_{\alpha}}{q_{\alpha}}\right)^{2}+r^{3}\left[\frac{1}{2} \dot{\varphi}^{2}-V(\varphi)\right]
$$


(accurate to complete derivative with respect to $t$ ). The relevant conjugate momentums are equal

$$
\begin{aligned}
p_{\varphi} & =\frac{\partial L}{\partial \dot{\varphi}}=r^{3} \dot{\varphi}, \quad p_{r}=\frac{\partial L}{\partial \dot{r}}=-6 r \dot{r} \\
p_{q_{\alpha}} & =\frac{\partial L}{\partial \dot{q}_{\alpha}}=r^{3} \frac{\dot{q}_{\alpha}}{q_{\alpha}^{2}}
\end{aligned}
$$

and the Hamiltonian of the system is

$$
H=p_{\varphi} \dot{\varphi}+p_{r} \dot{r}+\sum_{\alpha=1}^{3} p_{q_{\alpha}} \dot{q}_{\alpha}-L .
$$

Let's note, that for deriving the exact equations it was necessary to use the expression (田) for scalar curvature instead of $(\overline{0})$. Using the last one is impossible whereas in this one the integration for elimination of $\dot{q}_{\alpha} / q_{\alpha}$ is already yielded. It is intolerable as actually there is the deletion of variables $q_{\alpha}$ and thus truncation of the Hamiltonian. Let's note also, that if to use the last of relations (10) and expression $\dot{q}_{\alpha} / q_{\alpha}=C_{\alpha} / r^{3}$ from (5) exchanging simultaneously $p_{q_{\alpha}} \rightarrow \widehat{p}_{q_{\alpha}}$ (here $\widehat{p}_{q_{\alpha}}=-i \partial / \partial q_{\alpha}$ ), it is easy to obtain that $q_{\alpha} \widehat{p}_{q_{\alpha}} \Psi=C_{\alpha} \Psi$. The last means that in our case $\Psi$ is the eigenfunction of the operator $\widehat{p}_{q_{\alpha}}$. It allows with account (5) to write the Hamiltonian (11) as:

$$
H=\frac{1}{2} \frac{p_{\varphi}^{2}}{r^{3}}-\frac{p_{r}^{2}}{12 r}+r^{3} V(\varphi)+\left(\sum_{\alpha=1}^{3} C_{\alpha}^{2}\right) / 2 r^{3} .
$$

Quantizing (12) by replacement of momentums $p_{\varphi}$ and $p_{r}$ on $-i \partial / \partial \varphi$ and $-i \partial / \partial r$ accordingly and also using the rescaling $\varphi \rightarrow \sqrt{6} \Phi$ we obtain the Klein-Gordon equation

$$
\begin{gathered}
{\left[\frac{1}{r^{p}} \frac{\partial}{\partial r}\left(r^{p} \frac{\partial}{\partial r}\right)-\frac{1}{r^{2}} \frac{\partial^{2}}{\partial \Phi^{2}}-U_{e f}\right] \psi(r, \Phi)=0,} \\
U_{e f}=-6\left(\sum_{\alpha=1}^{3} C_{\alpha}^{2}\right) / r^{2}-12 r^{4} V(\Phi) .
\end{gathered}
$$

It is the required WDW equation in minisuperspace of the variables $r$ and $\Phi$. In this equation the parameter $p$ represents the ambiguity in the ordering of noncommuting operators $r$ and $p_{r}$. Let's emphasize, that the wave function of the Universe $\Psi$ does not depend on time. This circumstance is valid for the closed Universe by virtue of equality to zero of its total energy remains valid and for the flat Universe on the basis of given in Introduction reasonings. 
The transformation of the wave function

$$
\psi=r^{-p / 2} \Psi
$$

allows to eliminate first derivative in (13)

$$
\begin{gathered}
{\left[\frac{\partial^{2}}{\partial r^{2}}-\frac{1}{r^{2}} \frac{\partial^{2}}{\partial \Phi^{2}}-U_{e f}\right] \psi(r, \Phi)=0,} \\
U_{e f}=-\frac{p}{2}\left(1-\frac{p}{2}\right) \frac{1}{r^{2}}-\frac{6 \sum_{\alpha=1}^{3} C_{\alpha}^{2}}{r^{2}}-12 r^{4} V(\Phi) .
\end{gathered}
$$

Let's note, that in expression for an effective potential energy there is an addend of "centrifugal energy" $-p(1-p / 2) / 2 r^{2}$.

Is explored Eq. (14) with a various form of the potential $V(\Phi)$ and by choice of parameter $p$.

\section{A. Above barrier reflection of the wave function of the Universe}

1. de Sitter minisuperspace. Let's consider the simplest case of minisuperspace model, when the factor ordering $p=0$ and potential $V(\Phi)$ represents constant vacuum energy $\varepsilon_{v}$ creating an effective cosmological constant. Then WDW Eq. (14) will take the form of the one-dimensional Schrödinger equation

$$
\begin{gathered}
{\left[-\frac{d^{2}}{d r^{2}}+U_{e f}\right] \Psi(r)=0,} \\
U_{e f}=-6\left(\sum_{\alpha=1}^{3} C_{\alpha}^{2}\right) / r^{2}-H^{2} r^{4},
\end{gathered}
$$

where $H^{2}=12 \varepsilon_{v}$ is the Hubble parameter. Entering $\rho=H^{1 / 3} r$ and $\gamma=6 \sum_{\alpha=1}^{3} C_{\alpha}^{2}$ we can rewrite $(\mathbb{1 5})$ as

$$
\left[-\frac{d^{2}}{d \rho^{2}}+U_{e f}\right] \Psi(\rho)=0, \quad U_{e f}=-\gamma / \rho^{2}-\rho^{4} .
$$

Eq. (16) describes the motion of a "particle" with zero-point energy in the field of the effective potential $U_{e f}$. The interesting feature of the given potential is that near to the origin of coordinates it is approach infinity under the law $U_{e f} \approx-\gamma / \rho^{2}$ (that is we can neglect the second term in potential). As is known [9], this case 
is intermediate between when there are usual stationary states and the cases when happens the "collapse" of a particle in the origin of coordinates. Therefore, it is necessary to carry out the additional analysis here.

For this purpose we shall search near $\rho=0$ for solution of

$$
\left[\frac{d^{2}}{d \rho^{2}}+\frac{\gamma}{\rho^{2}}\right] \Psi(\rho)=0
$$

as $\Psi \sim \rho^{s}$ that at substitution in (17) gives:

$$
s^{2}-s+\gamma=0
$$

with roots

$$
s_{1}=1 / 2+\sqrt{1 / 4-\gamma}, \quad s_{2}=1 / 2-\sqrt{1 / 4-\gamma} .
$$

Further, we select around the origin of coordinates small area of radius $\rho_{0}$ and is exchanged in it the function $-\gamma / \rho^{2}$ by the constant $-\gamma / \rho_{0}^{2}$. Having defined wave functions in such "cut off" field then we shall look what happens at the passage to the limit $\rho_{0} \rightarrow 0$.

Let's assume at first that $\gamma<1 / 4$. Then $s_{1}>s_{2}>0$ and at $\rho>\rho_{0}$ the general solution of Eq. (17) looks like (at small $\rho$ )

$$
\Psi=A \rho^{s_{1}}+B \rho^{s_{2}}
$$

( $A, B$ are the constants). At $\rho<\rho_{0}$ the solution of

$$
\frac{d^{2} \Psi}{d \rho^{2}}+\frac{\gamma}{\rho^{2}} \Psi=0,
$$

finiteness in the origin of coordinates looks like

$$
\Psi=C \sin (k \rho), \quad k=\sqrt{\gamma} / \rho_{0} .
$$

At $\rho=\rho_{0}$ function $\Psi$ and its derivative should be continuous functions. Therefore, we can write one of requirements as the requirement of the continuity of the logarithmic derivative with respect to $\Psi$ that gives in

$$
\sqrt{\gamma} \operatorname{ctg} \sqrt{\gamma}=\frac{s_{1} \rho_{0}^{s_{1}-s_{2}}+(B / A) s_{2}}{\rho_{0}^{s_{1}-s_{2}}+(B / A)},
$$

or solving rather $(B / A)$ we obtain 


$$
B / A=\text { const } \rho_{0}^{s_{1}-s_{2}} .
$$

Passing on to the limit $\rho_{0} \rightarrow 0$ we find that $B / A \rightarrow 0$. Thus from two solutions (18) remains

$$
\Psi=A \rho^{s} .
$$

Let now $\gamma>1 / 4$. Then $s_{1}$ and $s_{2}$ are complex:

$$
s_{1}=-1 / 2+i \sqrt{\gamma-1 / 4}, \quad s_{2}=s_{1}^{*} .
$$

By analogy to the previous reasonings we again come to equality (19) which at substitution of values $s_{1}$ and $s_{2}$ gives

$$
B / A=\text { const } \rho_{0}^{i \sqrt{4 \gamma-1}} .
$$

At $\rho_{0} \rightarrow 0$ this expression does not approach to any limit so the direct passage to the limit $\rho_{0} \rightarrow 0$ is impossible. The general form of the real solution of (17) can be represented as following:

$$
\Psi=\text { const } \sqrt{\rho} \cos \left(\sqrt{\gamma-1 / 4} \ln \left(\rho / \rho_{0}\right)+\text { const }\right) .
$$

This function has zeros which number unlimited grows with decreasing of $\rho_{0}$. Then at any finite value of the total energy $E$ the "normal state" of a "particle" in given field corresponds to the energy $E=-\infty$. As "particle" is in infinitesimal area around of the origin of coordinates there is the "collapse" of the "particle" on centre.

Further, we find the vector of the probability density flux near to zero. In our one-dimensional case we have:

$$
\mathbf{j}=\frac{i}{2}\left(\Psi^{*} \frac{\partial \Psi}{\partial \rho}-\Psi \frac{\partial \Psi^{*}}{\partial \rho}\right)
$$

It is easy to obtain from here that: 1 ) in the case $\gamma<1 / 4$ at use $\Psi$ from (20) relevant probability density flux $\mathbf{j}=0$ (as well as for any real wave function). 2) At $\gamma>1 / 4$ with the account $\Psi$ from (22) we have $j=\mp \sqrt{\gamma-1 / 4}$. The upper sign corresponds to the ingoing and the lower one to the outgoing wave. The obtained result means that there is the constant probability density flux near to the origin of coordinates.

Thus we have two types of the behaviour of the wave function of a "particle" at various values of the parameter of an anisotropy $\gamma: 1$ ) at $\gamma<1 / 4$ the wave function near to the origin of coordinates tent to zero; 2 ) at $\gamma>1 / 4$ happens the "collapse" of a "particle" on centre. 


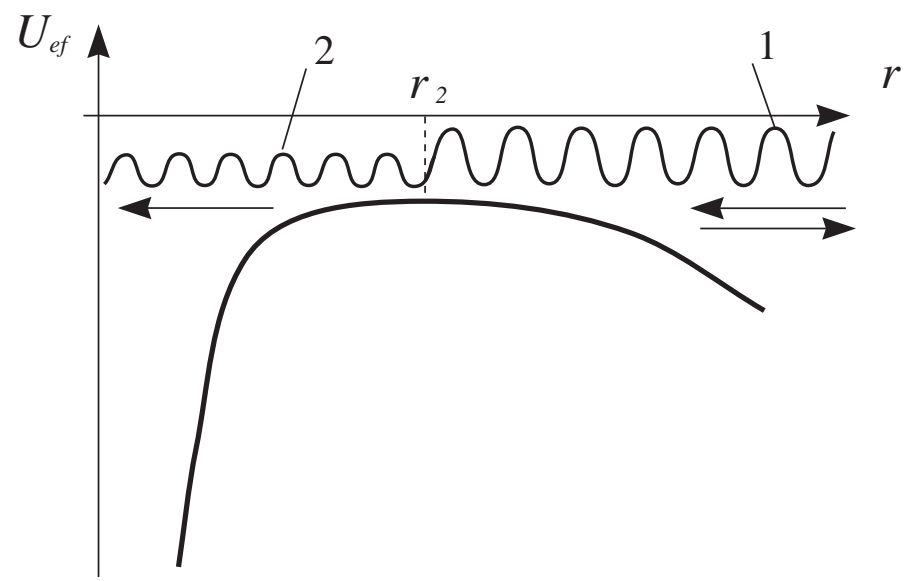

Figure 1: The above barrier reflection of wave function: 1 are ingoing and reflected waves; 2 is the passed wave.

Let's note that the probleme of falling of a particle on centre has been already studied in isotropic model with the matter equation of state $p=\epsilon$ in [10]. Let's consider further the motion of a "particle" in the semiclassical approximation. From the form of potential (16) and equality to zero of the total energy of the Universe follows that the examination of Eq. (16) is reduced to the one-dimensional problem about above barrier reflection (Fig. 1), i.e. to reflection of a "particle" with energy exceeding height of the barrier. In our case "particle" goes down to some "turning point" $\rho_{2}$ in which it changes the direction of motion on an inverse. The given point represents a complex solution of the equation $U_{e f}=0$, namely $\rho_{2}=\gamma^{1 / 6} \exp (i \pi(2 n+1) / 6)$. Then the required reflectance $R$ is found as [9]:

$$
R=\exp \left(-4 \operatorname{Im} \int_{\rho_{1}}^{\rho_{2}}\left[-U_{e f}\right]^{1 / 2}\right)
$$

(here $\rho_{1}$ is any point on the real axis). In the considered case using (16) and (23) we have:

$$
R=\exp \left(-\frac{2}{3} \sqrt{\gamma} \pi\right)
$$

The last expression is obtained with use of the semiclassical approximation. It is useful also to find the exact solution of Eq. (16) which looks like:

$$
\Psi(\rho)=C_{1} \sqrt{\rho} J\left(\frac{1}{3} \sqrt{1 / 4-\gamma}, \frac{1}{3} \rho^{3}\right)+C_{2} \sqrt{\rho} Y\left(\frac{1}{3} \sqrt{1 / 4-\gamma}, \frac{1}{3} \rho^{3}\right)
$$

where $C_{1}$ and $C_{2}$ are integration constants, $J(\nu, z), Y(\nu, z)$ - the Bessel functions of the first and second kind accordingly, $\nu, z$ are index and argument of these functions. For 
the finding of the reflectance it is necessary to consider the behaviour of this solution on major distances from the origin of coordinates. In this case $\Psi(\rho)$ describes ingoing and reflected waves and the reflectance will be equal to the ratio of amplitudes of these waves. The asymptotic form of the function $\Psi(\rho)$ at $\rho \rightarrow \infty$ is

$$
\Psi(\rho) \approx \sqrt{\frac{6}{\pi \rho^{2}}} \cos \left(\frac{1}{3} \rho^{3}-\frac{1}{6} \pi \sqrt{1 / 4-\gamma}-\frac{\pi}{4}\right) .
$$

It is clear from here that:

$$
\begin{gathered}
\text { for } \gamma<1 / 4: \quad R=1 \\
\text { for } \gamma>1 / 4: \quad R=\exp \left(-\frac{2}{3} \pi \sqrt{\gamma-1 / 4}\right) .
\end{gathered}
$$

The second expression from (27) at $\gamma \gg 1 / 4$ coincides with reflectance from the semiclassical approximation (24).

Being based on results obtained at the analysis of behaviour of the wave function of the Universe near to the origin of coordinates is concluded that at $\gamma<1 / 4$ does not happen the accumulation of the wave function at $\rho \rightarrow 0$ and consequently takes place the complete reflection of the wave function from the barrier $(R=1)$. In the case $\gamma>1 / 4$ which corresponds to collapse of a "particle" on centre the reflectance $R$ becomes less than 1 . It happens because there is nonzero probability density flux in the infinitesimal area around of the origin of coordinates. Let's note that at the approach to zero the problem, generally speaking, ceases to be stationary. It gives that the wave function can accumulate in this area.

2. Variable scalar field. Let's consider further the case of Eq. (14) when the factor ordering $p$ is equal to zero as before, but potential of the scalar field is variable: $V(\varphi)=m^{2} \varphi^{2} / 2$. Introducing the rescaling $\varphi \rightarrow \sqrt{6} \Phi$ and $m \rightarrow \mu / 6$ we shall write the WDW equation as:

$$
\begin{gathered}
{\left[-\frac{\partial^{2}}{\partial r^{2}}+\frac{1}{r^{2}} \frac{\partial^{2}}{\partial \Phi^{2}}+U_{e f}\right] \Psi=0} \\
U_{e f}=-\frac{\gamma}{r^{2}}-\mu^{2} r^{4} \Phi^{2} .
\end{gathered}
$$

The finding of the analytical solution of the obtained equation represents a complex problem. Therefore, for its examination we shall search the WKB solution as $\Psi_{c}=$ $e^{-i S}$. The relevant equation for action $S(r, \Phi)$ is

$$
-\left(\frac{\partial S}{\partial r}\right)^{2}+\frac{1}{r^{2}}\left(\frac{\partial S}{\partial \Phi}\right)^{2}-U_{e f}=0
$$


For finding of the solution of this nonlinear differential equation it is possible to reduce it to system of the ordinary differential equations called the characteristic system of the given partial equation. Using this system it is possible to construct an integrated surface of Eq. (29), consisting from the characteristics. The required system of the characteristics written with respect to arbitrary parameter $t$ has a form [11] :

$$
\begin{aligned}
r^{\prime}(t) & =-2 p, \quad \Phi^{\prime}(t)=\frac{2}{r^{2}} q, \\
S^{\prime}(t) & =-2\left(\frac{\gamma}{r^{2}}+\mu^{2} r^{4} \Phi^{2}\right), \\
p^{\prime}(t) & =\frac{2}{r^{3}} q^{2}+2 \frac{\gamma}{r^{3}}-4 \mu^{2} r^{3} \Phi^{2}, \\
q^{\prime}(t) & =-2 \mu^{2} r^{4} \Phi,
\end{aligned}
$$

where ' denote a derivative with respect to $t$ and introducing denotations $p=\partial S / \partial r$, $q=\partial S / \partial \Phi$. The obtained system of equations describes an one-dimensional motion of a "particle" along the characteristic. In this case monotonically varying parameter $t$ can play a role of time [12] in due course there is an evolution of the Universe.

The obtained system of characteristics can be use for finding of dependence of coefficient of above barrier reflection $R$ of wave function of the Universe from value of the field $\Phi$.

But before we shall note one useful possibility of simplification of making of a calculation. Expand an effective potential $U_{\text {ef }}$ from (16) near to a maximum in a Taylor series:

$$
U_{e f}=U_{\max }+\frac{\alpha}{2}\left(\rho-\rho_{\max }\right)^{2},
$$

here $U_{\max }$ and $\rho_{\max }$ are a maximal value of potential and relevant value of $\rho$, and $\alpha$ is a value of a second derivative with respect to $U_{\text {ef }}$ from (16) in the point $\rho_{\max }$. The values of specified quantities are easily finding from (16):

$$
\begin{gathered}
U_{\max }=-3\left(\frac{\gamma}{2}\right)^{2 / 3}, \rho_{\max }=\left(\frac{\gamma}{2}\right)^{1 / 6} \\
\alpha=-24\left(\frac{\gamma}{2}\right)^{1 / 3} \cdot
\end{gathered}
$$

Then using (23) and (31) we have

$$
R=\exp \left(-\frac{1}{2} \sqrt{\frac{3}{2}} \sqrt{\gamma} \pi\right)
$$




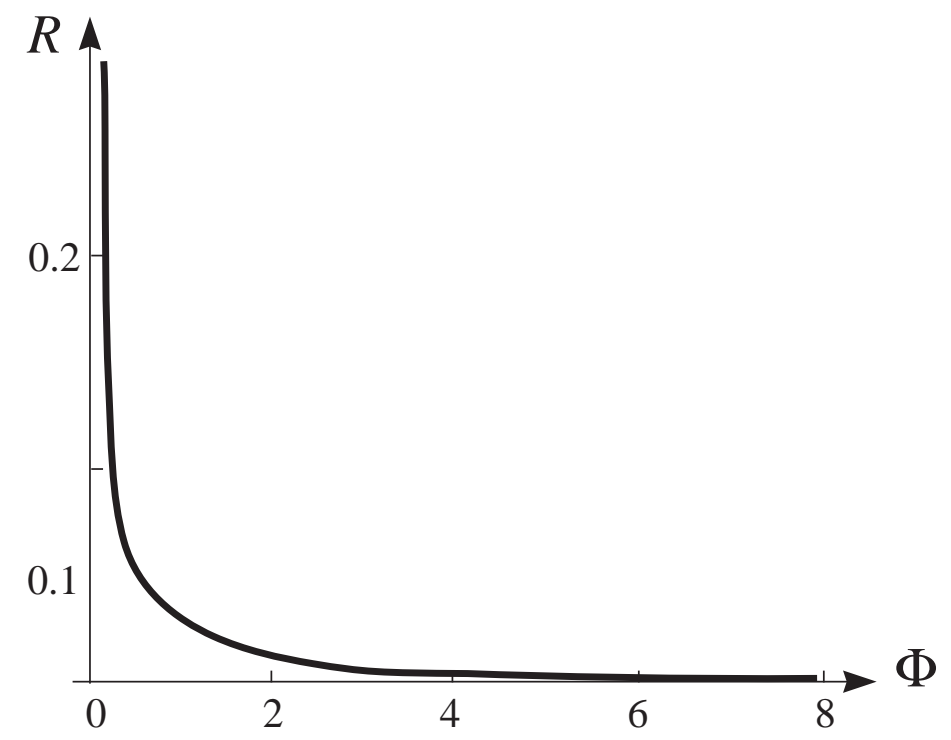

Figure 2: Dependence of coefficient of above barrier reflection $R$ from value of the scalar field $\Phi$.

that approximately coincides with (24). Thus in an one-dimensional case there is an possibility to use approximate expression (31) instead of exact one.

Further, we shall search for the solution of system (30) numerically. Using (23) and (31) it is possible to obtain the following form of dependence $R(\Phi)$ (Fig. 2). It is obvious that the reflectivity promptly decreases with increasing of value of the field $\Phi$. After reflection of the wave function the following necessary stage of evolution of the Universe is the inflationary period providing the "stretch" of linear size of the Universe with Planck up to macroscopic. For ensuring of the sufficiently long period of inflation it is necessary the realization of two requirements on scalar field (see e.g. [13] ): 1) it should be a Planck order; 2) its values should vary slowly with time. The realization first of these requirements corresponds rather small, but nevertheless distinct from zero reflectivity $R$ (see Fig. 2). Solving system (30) will be easily convinced that is fulfilled second of the posed requirements also.

\section{B. The wave function bouncing off a potential wall}

Now we shall consider Eq. (14) in a case when the parameter $p$ is different from zero and there is a potential of the scalar field $V(\varphi)=m^{2} \varphi^{2} / 2$. At such setting of a problem we have two essentially various variant of the effective potential $U_{e f}$. At realization of a requirement $0 \leq p \leq 2$ the form of $U_{\text {ef }}$ from (14) as a matter of fact by nothing differs from the case when $p=0$ and the relevant consideration 


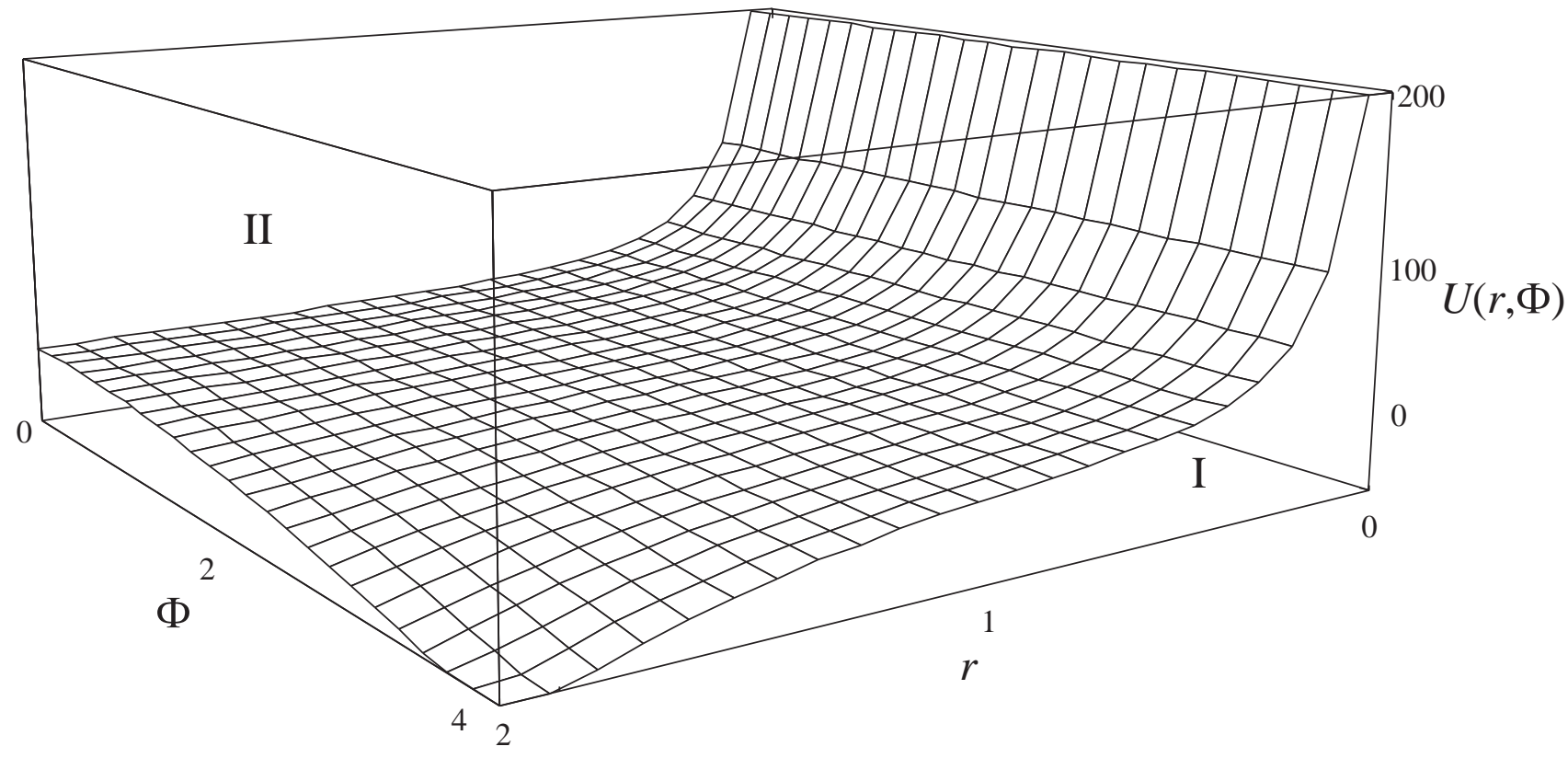

Figure 3: The effective potential $U_{\text {ef }}$. I is a classically forbidden area, II is a classically allowed area.

will be made by analogy to the problem studied in the previous section. In a case omissions of the mentioned above requirement the qualitatively new statement of a problem is possible. For this purpose the realization of one requirement is necessary: $\left|-p^{2} / 2+p / 2\right|>\gamma$, which ensures occurrence in the effective potential of a positive factor before $1 / r^{2}$ greater than $\gamma$. Thus there is a possibility of occurrence of a repelling potential wall. Then the effective potential in (14) will be:

$$
U_{e f}=\frac{\left|-p^{2} / 2+p / 2\right|}{r^{2}}-\frac{\gamma}{r^{2}}-\mu^{2} r^{4} \Phi^{2}-\varepsilon .
$$

Thus the influence of a massless scalar field (e. g. photon gas) with energy density $\varepsilon$ also is taken into account, the sense of which introduction will be explained below. The form of potential is shown on Fig.3. Then Eq. (14) describes a motion of a "particle" with a zero-point energy in potential $U_{e f}(r, \Phi)$.

As is obvious from Fig.3 the effective potential parts space on two areas: classically forbidden (interior) and classically allowed (exterior). At transition of a "particle" in the I-st area its wave function will be exponentially decay (as $U_{e f}(r, \Phi)$ approach infinity at $r \rightarrow \infty$ ) and the relevant probability of realization of the given state tends to zero.

On the other hand, the wave function $\Psi$ describes the wave incident on the barrier 
$U_{e f}(r, \Phi)$ on the part of major $r$ and the wave reflex from the barrier. The physical sense of the given statement consists that the incident wave describes the contracting Universe and reflex - expanding one.

We shall consider an evolution of the scalar field $\Phi$ at stages of contraction and expansion. For this purpose we shall search the WKB solution of the equation (14) with potential (33) in classically allowed range as $\Psi_{c}=e^{-i S}$. The relevant equation for action $S(r, \Phi)$ will be:

$$
-\left(\frac{\partial S}{\partial r}\right)^{2}+\frac{1}{r^{2}}\left(\frac{\partial S}{\partial \Phi}\right)^{2}-U_{e f}=0 .
$$

By analogy to the case of Eq. (29) we shall make system of the characteristics with respect to arbitrary parameter $t$ :

$$
\begin{aligned}
r^{\prime}(t) & =-2 p, \Phi^{\prime}(t)=\frac{2}{r^{2}} q, \\
S^{\prime}(t) & =2\left(\frac{\left|-p^{2} / 2+p / 2\right|}{r^{2}}-\frac{\gamma}{r^{2}}-\mu^{2} r^{4} \Phi^{2}-\varepsilon\right), \\
p^{\prime}(t) & =\frac{2}{r^{3}} q^{2}-2 \frac{\left|-p^{2} / 2+p / 2\right|}{r^{3}}+2 \frac{\gamma}{r^{3}}-4 \mu^{2} r^{3} \Phi^{2}, \\
q^{\prime}(t) & =-2 \mu^{2} r^{4} \Phi .
\end{aligned}
$$

As well as in case of Eq. (29) system of equations (35) is describes an one-dimensional motion of a "particle" along the characteristic. Thus the behaviour of a "particle" is similar on bounce off a potential barrier $\Phi(r)$ made at cross of the effective potential with the plane $\Phi-r$ (that is when $U_{e f}=0$ ). Therefore, a semiclassical wave function $\Psi_{c}$ describes an ensemble of classical universes evolving along the characteristics $S$. Then the ensemble of these characteristics can be considered as the trajectories of a motion with the various initial conditions.

We shall note here that introduced earlier the massless scalar field with the energy density $\varepsilon$ is necessary that the Universe having a zero total energy always remained in classically allowed area (Fig. 4).

As it was specified above the evolution of the Universe is described by two stages: at first regime of contracting and then, after bounce off, regime of expansion. At the stage of contracting the field makes oscillations with increasing amplitude. After bounce off two variants are possible: 1) the field $\Phi$ amount to rather major value and after reflection varies slowly that corresponds to an inflationary period and further transfers on scalaron stage (Fig. 5); 2) $\Phi$ hasn't amount to major values and having reflected at once makes fast oscillations losing the energy. Thus, naturally, there is no inflationary stage (or it is too short). 


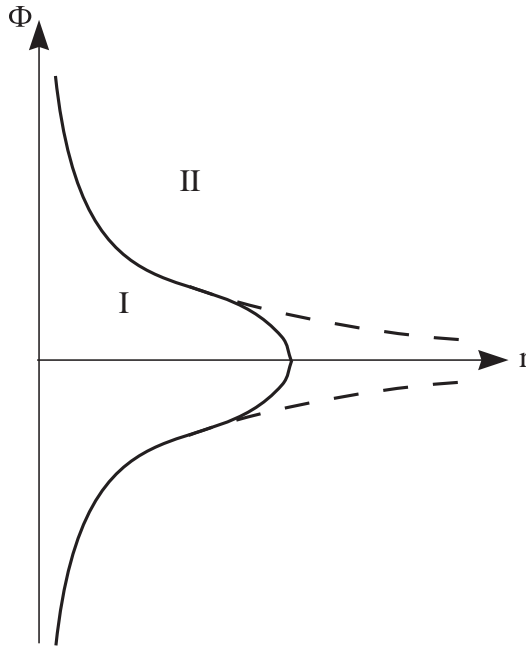

Figure 4. Conventional form of the potential barrier with $\varepsilon . \quad I$ is a classically forbidden area; II is a classically allowed area. The dashed lines - form of the barrier without $\varepsilon$.

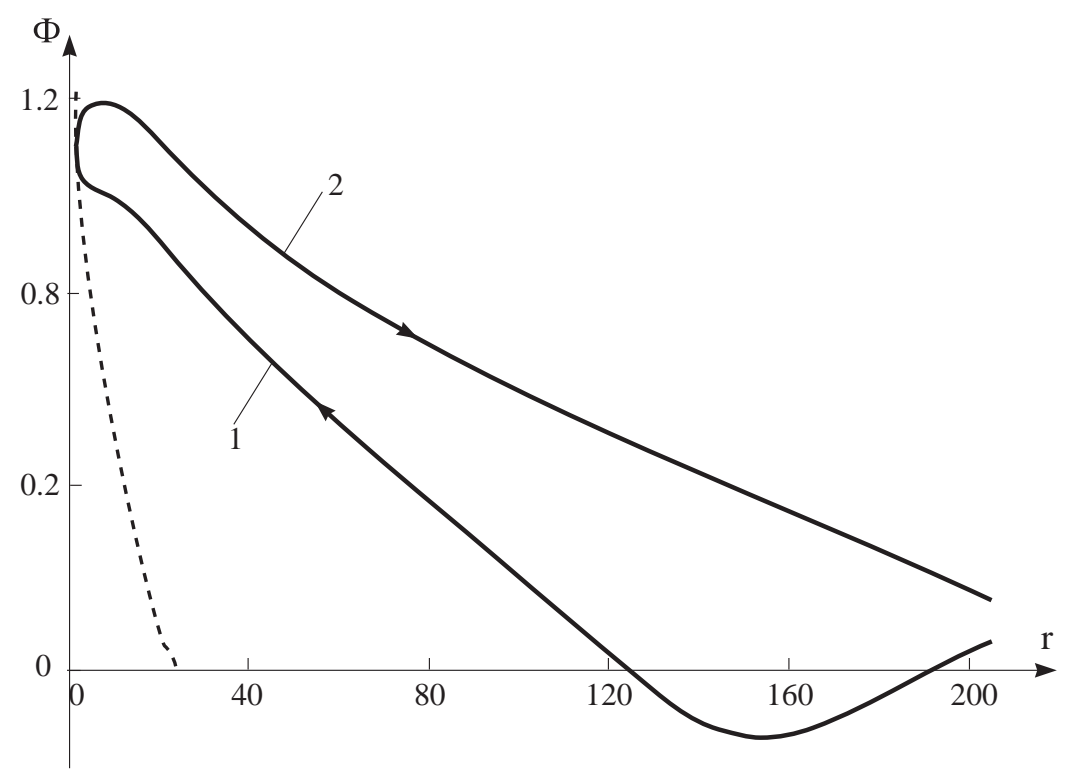

Figure 5. The diagram of dependence of the scalar field $\Phi$ from $r .1$ is an evolution of the field on the contracting stage, 2 - during the inflation. The dashed line conventionally shows the form of the potential barrier.

It is necessary apart to note that in considered model the presence of a repelling potential wall does not allow the Universe to collapse.

\section{Conclusions}

In the submitted paper we have considered an anisotropic cosmological Bianchi type I model with the scalar field. Distinctive feature at the solution of the given problem was representation of three scale factors in such manner that the final Einstein's equations have turned out dependent only from one function $r(t)$.

In the quantum approach the basic equation of the quantum cosmology - WDW equation (13) was obtained. The examination of the latter was reduced to considering the following problems:

1. The simplest model with constant scalar field playing a role of an effective cosmological constant was studied. It has allowed to reduce the WDW equation to the one-dimensional Schrödinger equation. The presence of the parameter of an anisotropy $\gamma$ in the effective potential gives in interesting feature: there is some critical value of this parameter at which there is partitioning the problem into two variants. In the first case at $\gamma>1 / 4$ the collapse of the wave function on centre takes place. Thus there is the constant nonzero probability density flux $j$ that means an possibility of accumulation of the wave function in close to the origin of coordinates area. In the second case $(\gamma<1 / 4)$ the collapse of the "particle" on centre misses and $j=0$. 
Further, the problem of finding of coefficient of above barrier reflection $R$ from the cosmological singularity of the wave function of the Universe $\Psi$ was solved. For this purpose two approaches were used: 1) semiclassical approximation and 2) finding $R$ as the ratio of amplitudes of wave functions of reflected and ingoing waves on infinity. Both approaches give identical results at $\gamma \gg 1 / 4$. Thus $R<1$ that means partial penetration of the wave function into close to zero area and its further collapse on centre. In case of $\gamma<1 / 4$ the second approach gives $R=1$ as against semiclassical one. It speaks about an inapplicability the latter in such situation and about necessity to use asymptoticses of the exact solutions.

Let's note that on coefficient of above barrier reflection the influence of Hubble parameter has not an effect in any way because it is only renormalaze the scale factor.

2. The model with a variable scalar field with potential $V(\varphi)=m^{2} \varphi^{2} / 2$ was considered. In view of complexity of finding of an exact analytical solution the given problem was explored numerically. With this purpose the semiclassical solution of Eq. (28) was found, therefore Eq. (29) is obtained for which the system of the characteristics (30) was obtained. The last one was used for finding of relationship of the coefficient of the above barrier reflection $R$ from value of the field $\Phi$. The obtained relationship shows that $R$ is rather great at Planck field. It means that after reflection there is an possibility of an output of the Universe on a rather long inflationary stage providing increase of its size with Planck up to macroscopic and a further output on the standard cosmological scenario.

3. In case of nonzero factor ordering there is essentially new possibility of a "bounce off" a wave function of the Universe from a repelling potential wall ensured with a form of effective potential (33). By analogy to the previous case the solution of Eq. (34) was found with the help of system of the characteristics (35). The obtained results show that the Universe beginning the evolution with small initial value of $\Phi$ at the stage of contraction gathers a field up to Planck. After bounce off the field will increase still and further Universe transfers to an inflationary stage (see Fig. 5).

Note that in considered cases above barrier reflection and bounce of a wave function of the Universe feature process of the quantum creation of the Universe. The further evolution of the model represents a stage of expansion with prompt losses of an anisotropy and transition into Friedmann Universe.

\section{Acknowledgements}

We are grateful to A.A. Starobinsky and I.B. Khriplovich for useful discussions of results.

This work was supported by the research grant KR-154 of International Science and Technology Centre (ISTC). 


\section{References}

[1] B. S. DeWitt, Phys. Rev. 160, 1113 (1967).

[2] Ya. B. Zeldovich, Sov. Astron. Lett. 7, 322 (1981); A. Vilenkin, Phys. Lett. B 117, 25 (1982).

[3] R.Rajaraman, "Solitons and Instantons. An Introduction to Solitons and Instantons in Quantum Field Theory", North - Holland Publishing Company, Amsterdam - NewYork - Oxford, 1982.

[4] S.W. Hawking and N. Turok, Phys. Lett. B 425, 25, (1998).

[5] A. D. Dolgov, Ya. B. Zeldovich, M. V. Sazhin "Cosmology of the Early Universe", Moscow University Press, Moscow, 1988.

[6] S. Coleman and F. D. Luccia, Phys. Rev. D 21, 3305 (1980).

[7] J. R. Gott and T. S. Statler, Phys. Lett. B 136, 157 (1984).

[8] V. Ts. Gurovich and A. A. Starobinsky, Sov. Phys. JETP 50 , No. 5, 844 (1979).

[9] L. D. Landau and E. M. Lifshitz, Quantum Mechanics, Pergamon, Oxford, 1965.

[10] M.L. Fil'chenkov, Phys. Lett. B 354 , 208 (1995).

[11] V. Ts. Gurovich U. M. Imanaliev and I. V. Tokareva, JETP Lett. 64 , No. 5, 329 (1996).

[12] A. Vilenkin, Texas Rel. Astrophys., 271 (1992); "Quantum Cosmology", gr-qc/9302016.

[13] A. D. Linde, "Elementary Particle Physics and Inflationary Cosmology", Nauka, Main Editorial Board for Physical and Mathematical Literature, Moscow, 1990. 